\title{
The Art of Human Intelligence and the Technology of Artificial Intelligence: Artificial Intelligence Visual Art Research
}

\author{
Feng Tao ${ }^{1[0000-0001-7967-2544]}$; Xiaohui Zou ${ }^{20000-0002-5577-8245]}$; Danni Ren ${ }^{3}$ \\ School of Philosophy, Nankai University, Tianjin 300071; \\ philart@163.com \\ ${ }^{2}$ Sino-American Saerle Research Center; \\ 949309225 @qq. com \\ ${ }^{3}$ College of Foreign Languages, Nankai University, Tianjin 300071; \\ rendanni@139.com
}

\begin{abstract}
This article aims to find out how human intelligence and artificial intelligence can complement each other better through the comparison of art and technology. Here is an example of artificial intelligence visual art research. The method is: First of all, from the characteristics of artistic language, explore the charm of human intelligence. Furthermore, from the characteristics of technical language, we can feel the power of artificial intelligence. Finally, from the characteristics of human-computer interaction and typical examples, we can explore how humanities and engineering complements each other. In this paper, we discuss the creativity, artistic standards and artificial reception issues in artificial intelligence art through analyzing the latest artificial intelligence art programs such as "Creative Adversarial Networks" (CAN) and Google Deep Dream. We argue that creativity is not only novelty, such as "style ambiguity" and other techniques, but also originality. The result is the discovery of that the art standard cannot be limited to be searched in previous works of art. The art standard itself is historical and changeable, and is the "true content". Both Creativity and art standard need us comprehend and rethink about the overall laws and paradigms of whole art. However, from the perspective of art reception, the artificial intelligence art has its own unique value. Its significance lies in: it has opened up a vast new world for the intelligence art education. Further, we can also compare the thinking habits of artists and scientists and engineering technologists respectively from the linguistic cognition. Next, we will study that the cooperative research topic of digital and textual chessboard combined with human-computer will not only help teachers and students in the art education process build their own personalized knowledge bases. It would be a better understanding of the benefits of combining human brain and computer.
\end{abstract}

Keywords: Artificial Intelligence Art, Creativity, Aesthetic Reception

Can machine draw pictures? Can artificial intelligence create artworks? Now it seems that this is no longer a problem. Some intelligent programs such as CAN ("Creative 
Adversarial Networks") system and Google Deep Dream have been able to create works of art.

Computer art is the foundation and precursor of artificial intelligence art. Since the 1990s, with the breakthrough of some key artificial intelligence technologies, artificial intelligence art has gradually separated from traditional computer art and may become a new art form. Artificial intelligence art pays more attention to the intelligence or autonomy of computers. The fundamental purpose is to automatize computers, which is to simulate human intelligence to create artworks through programs, web search, and Deep Learning. And these artworks can be understood and appreciated by human. The study of artificial intelligence art helps to improve the creativity, imagination and emotional perception that computers did not have before. At the same time, it is also an inquiry into the principles of relevant parts of human intelligence. Artificial intelligence visual art is a combination of artificial intelligence technology and art. It not only is closely related to the technologies of artificial intelligence recognition, image processing, drawing, etc. but also involves the re-recognition of artworks and the re-definition of human artistic behaviors.

But is artificial intelligence really able to create works of art? What is the principles and mechanisms of this process, and how can artificial intelligence be creative? Does artificial intelligence solve the problem of art standards? How should we look at these works of art?

\section{Introduction}

Before answering these questions, let's reexamine the meaning of art. Since ancient times, thinkers have made many definitions of art, but the connotation and extension of art are constantly changing. In ancient Greece, art was regarded as a skill of man, including crafts and art. In the Age of Enlightenment, the scope of art was reduced to free art such as painting, literature, music, etc., and in the Internet age, the scope of art has been expanded significantly, such as photography, movie, computer art, new media art, etc. Looking at the definitions of art, it is basically believed that art is a unique behavior of human beings, and art is a work of human beings. Therefore, the philosopher Hegel distinguished between artistic beauty and natural beauty, and limited the objects of aesthetics into artistic beauty. The art theorist E. Gombrich directly claimed: "There really is no such thing as Art. There are only artists." [1] According to this, machine is not human, and thus the works it creates cannot be called art. But if we look at the results of the action, the intelligence agents can indeed produce some works that can be understood and appreciated by human beings and bring joy to people. Therefore, when artificial intelligence can also create works, it seems that we need to redefine the art. At this time, we can divide the artificial intelligence art problem into two parts. One is the internal problem: it involves some problems like the subject of artistic creation, subjectivity and intentionality, as well as aesthetic emotions. The other can be called an external problem: research from the results of behaviors, such as the work 
itself, the aesthetic effect of the work, etc. In fact, these two problems cannot be completely divided. However, we can temporarily suspend the subject of maker firstly, and only analyze it from the phenomenon and results. We can check if the CAN system is really creative, what the artistic standards used in the evaluation of artificial intelligence creativity by scientists, and the meaning of artificial intelligence art from the perspective of aesthetic acceptance.

At the beginning of the 21 st century, with the development of neural networks and Deep Learning techniques, artificial intelligence art makes a breakthrough in imitating human creativity and imagination. E.g., the University of Tübingen uses neural network algorithms to combine realistic images with artist styles to produce artistically styled images [2]. In 2016, Google Deep Dream (GDD) made use of neural network technique to train machines to learn to recognize images and further generate artistic images. The scientists created the GAN program (Generative Adversarial Networks) to simulate and generate similar works by making computers learn and imitate classic works in art history. However, this still cannot allow the computer to get rid of the suspicion of copying works. In 2017, scientists further created the CAN (Creative Adversarial Networks) program, a computer program that, based on the original GAN, modifies the network's goals to deviate from the established style as much as possible. At the same time, it tries to stay within the scope of the artwork but create creative images. This kind of program makes the computer no longer simply copy the model, but by emulating the creative activities of human beings, it can create a style of art independently. According to the double-blind test conducted by art lovers and experts, people can be deceived by the paintings created by the CAN program successfully, that is, the computer successfully passed the Turing test, and it seems to have really been intelligent in visual art [3].

Let us briefly summarize the working principles and mechanisms of the current artificial intelligence visual art. Firstly, the algorithms and programs of the computer are the foundation. Any drawing and design, visual recognition, generation programs, identification standards, etc. required in the artificial intelligence art must be formalized, that is, represented by algorithms. Secondly, the expert database and network data can provide sufficient information. Take the CAN program as an example. It needs to provide the intelligent agents with enough works of art to learn, imitate and identify. Thirdly, whether it is GDD, GAN or CAN program, it is based on Artificial Neural Networks. The so-called artificial neural network is "a model based on the human brain" [4]. By imitating the working mechanism of human neurons, it can make the machine self-improve and learn. The most important point is the Deep Learning method developed in the last decade, which refers to letting computers learn from experience and understand the world according to the concept of layering [5]. One of the breakthroughs in Deep Learning is to strengthen learning, that is, to learn to perform tasks by trial and error without being supervised. It is these innovations of key technologies that have made AI visual art a breakthrough. 


\section{Related Work}

\subsection{Creativity in $\mathrm{AI}$ art}

Some scholars believe that creativity is necessary for human progress. The famous artificial intelligence philosopher M. Boden says: "Creativity is a fundamental feature of human intelligence, and an inescapable challenge for AI." [6] Art is an embodiment of human creative thinking, and creative thinking is an ability of human intelligence that is the most complex and difficult to be formalized. So what is the creativity in art? Can it be formalized? Can artificial intelligence have a certain degree of creativity?

AI experts believe that intelligent agents can be given a certain degree of creativity through designing programs and Deep Learning skill. Turing refuted the so-called computer's rules obedience and inability to create as early as 1950 . He suggested the socalled originals rooted in the "education" of people, they are perhaps the result of the influence of some famous universal rules [7].

Boden suggests that creativity can be defined and formalized, and be represented by algorithms. She divides creativity into "Improbabilist" creativity and "Impossibilist" creativity [8]. The former is a recombination of various ideas, while the latter is to create new ideas that have never appeared before. Boden believes that Cohen's AARON program can implement some bionic one-arm operations, and when the generating program incorporates evaluation criteria such as aesthetic balance, it can decide what to do next by thinking about its behaviors. This has been somewhat creative, but AARON still can't reflect on its works, and accordingly adjust to improve the quality.

The author believes that creativity can be divided into two types, one is "novelty" and the other is "originality". The novelty is "from birth", that is, Boden's " Improbabilist " creativity, while the originality is Kant's genius, " Impossibilist creativity", and "ex nihilo"(creation out of nothing). And this is the originality with the exemplary meaning pursued by the artists.

Let's take a look at the creative part of CAN. Above all, we must know that the development of CAN and GAN (Generative Adversarial Networks) is the result of the development of artificial neural networks and Deep Learning techniques. Both gain the ability to generate artistic images after learning a large number of works of art in history. The biggest difference between CAN and GAN is that GAN can only imitate, and CAN generate "creative art by maximizing deviation from established styles and minimizing deviation from art distribution" [3]. The GAN system has two confrontational programs: generator and discriminator. The computer learns to generate new images through the generator, and the discriminator is responsible for judging the artistic images that match the art training section set by the computer. In this case, it is impossible to generate new and creative images. The novelty of CAN is that it sets two standards in the discriminator, one is "whether it is art" and the other is "what style of art is generated". These two standards are set to be confrontational, that is, it is necessary to generate images belonging to the art category, and to set images that are different from the established style, that is, an ambiguous artistic style. 
We can see that the CAN program is innovative, using stylistic ambiguity (i.e. maximal deviation from the established style) and identifying artistic standards (minimum deviation from the artistic scope). In other words, innovation is a breakthrough and deviation from the original rules within the established scope. CAN programmers believe that the reason why art needs innovation is that art itself has the property of "external stimulus patterns". People will get used to external stimuli. "a moderate arousal potential" can help people wake up aesthetic sense, too little stimulation can be boring and too much can be disgusting. Therefore, the designer's task is to try to "increase the stylistic ambiguity and deviations from style norms, while at the same time, avoiding moving too far away from what is accepted as art." [3] The CAN programmers made five groups double-blind experiments. Art lovers and art history students were invited to compare abstract expressionist paintings, Basel art exhibitions, CAN paintings, and GAN paintings. The results showed that most people think that CAN paintings are artworks and have some certain artistic characteristics like intentions, visual structures, communicability, etc.

Now, let's briefly compare the high-score artwork with the artworks of V. Kandinsky and W. Turner.

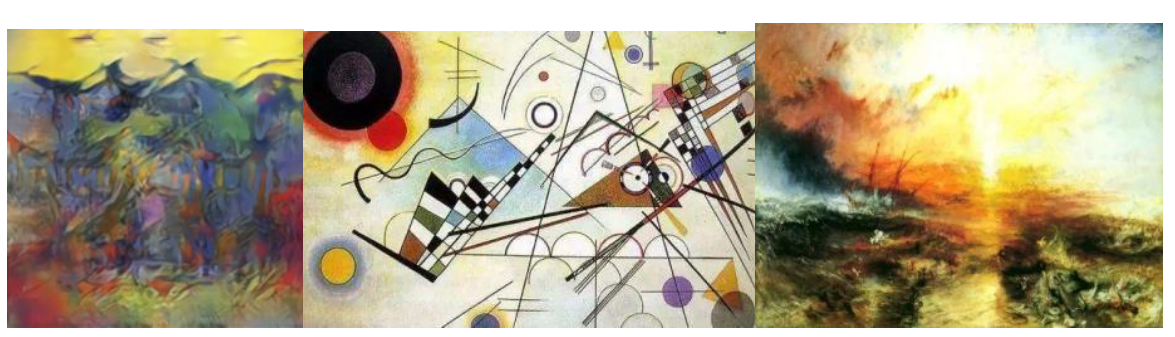

Fig. 1

Fig.2

Fig.3 (from left to right)

Fig.1: CAN high-score work, ranked first in audience preferences, ranked second in terms of human works and intentions. [3]Fig.2: V. Kandinsky, composition viii (cited from internet).Fig.3: W. Turner, The Slave Ship (cited from internet) .

From the author's point of view, the CAN work seems to be less harmonious in color than the other two artists' works. It may be due to too much dependence on calculations, making the colors more turbid and mediocre (see Fig.1). The composition and shape are also a little unbalanced, unlike Kandinsky's work, which takes into account the relationship between shape, sound and color (see Fig.2). When we look at Turner's work, it seems that the cloud and wind are unshaped and ambiguous, however the composition of picture is perfect, and significant (see Fig.3). The author believes that although the CAN work seems to be novel, it is not a superior work. It is disharmony and unbalanced in aesthetic whether its color, shape, composition or brush strokes. Thus, there is no aesthetic logic.

The reason why there is such deviation in judgment, the author believes, is that, first of all, creativity is not only a "novelty" or difference, but what is more important is in its "exemplary" meaning. Artists pursue "originality", that is to say, it involves more fundamental issues in art and innovations in the paradigm of thinking. And the means of innovation are also instructive. For example, the problem of the intuitiveness of the color needs impressionists to solve and modernist painters consider the problem of the 
planar authenticity of painting. Intelligent agents can't predict the direction of the essence of art from the known works of art history, let alone the innovation means of solving the problem. Computer analysis and induction of data cannot be raised into problem consciousness and thought, which is the fundamental problem of artificial intelligence innovation. Currently the mechanic of AI art is programmatic reorganization and piecing together the original images. Even if its works could be novel, it cannot be considered as success, let alone original. In addition, whether it is CAN, GAN, or other art programming, we must set a judging standard (i.e. evaluation system) [9], such as the art identification, the style identification, and the art lovers' marks in the test set in the CAN program. How are these standards determined? Can you really judge whether artificial intelligence works are real artworks and have creativity? This involves the issue of art standards. Is there any standard in art? Is the art standard fixed and unchangeable? Is the art standard subjective or objective? Next, let's briefly discuss the issue of art standards.

\subsection{Art standard in $\mathrm{AI}$ art}

From the logical operations, to the evaluation system, to the "trial and error method" in Deep Learning, all involve standards, especially the standards based on true and false. Some experts believe that aesthetic and artistic activities can be formalized. The premise should be that art works become the "prime objects for aesthetic evaluation" [10]. In addition, the audience's reaction and evaluation can be quantified. But at the same time, artificial intelligence experts admit that art evaluation is very subjective, so computers may not be able to evaluate their "own creative efforts in human terms" [11].

When artificial intelligence visual art generates a work, it must first judge whether it is a work of art. So how could it judge? When designing the art criticism algorithms and design algorithms, Gips and Stiny coded "conventions" and "criteria" as an aesthetic system, and then input the object to be evaluated to see if it conforms to the system [9]. We can see that the computer setting the evaluation criteria still depends on some previous evaluation criteria. Therefore, choosing different evaluation criteria will inevitably cause deviation from the evaluation target. For example, Duchamp's work "Spring" cannot be considered as a work of art according to any previous artistic standard. However, we know that "Spring" as groundbreaking ready-made art has greatly expanded the boundary of art.

In the CAN system, AI classifies some similar styles of artworks by deep learning in classic works in art history and sums up the general characteristics, then it can make a certain standard. But how to judge whether a new work is an artwork, faces the contradiction between innovation and standards. The CAN system claims that if the aesthetic consciousness of the audience is excessively awakened, it will cause the audience to be disgusted. For example, the work of the GDD program is excessively awakening, thus causing a negative evaluation of the critics [3]. But we compare GDD works with many modernist works. The former seems to be much milder. That is to say, the works of the Fauvism, Expressionism, Cubism and other genres are excessively awakening according to the evaluation standard of CAN. They should be excluded from the art system. 
Therefore, we can see that no matter how artificial intelligence is trained, its foundation is the existing works, and the artistic standards obtained by quantifying and analyzing the previous works are in conflict with the essence of the continuous innovation of modern art. Since art is an ever-developing living body, artistic standards are constantly changing. Adorno proposed a standard for judging true art and cultural goods as "truth content" ([Germany] Wahrheitgestalt), [12]. The truth content is different from the true and false binary logic of computer, but is the artistic standard that has both true and false and specific content. The language of art is real, which means that it truly simulates the language of things, and truly formalizes the language logic of itself, and the language logic of art is internal and historical [13]. According to Adorno, the artistic standard is a large standard based on human imitation and true language. It can be regarded as the standard of the whole human artistic spirit. Therefore, the standards that do not take into account the evolution of artistic logic are rigid.

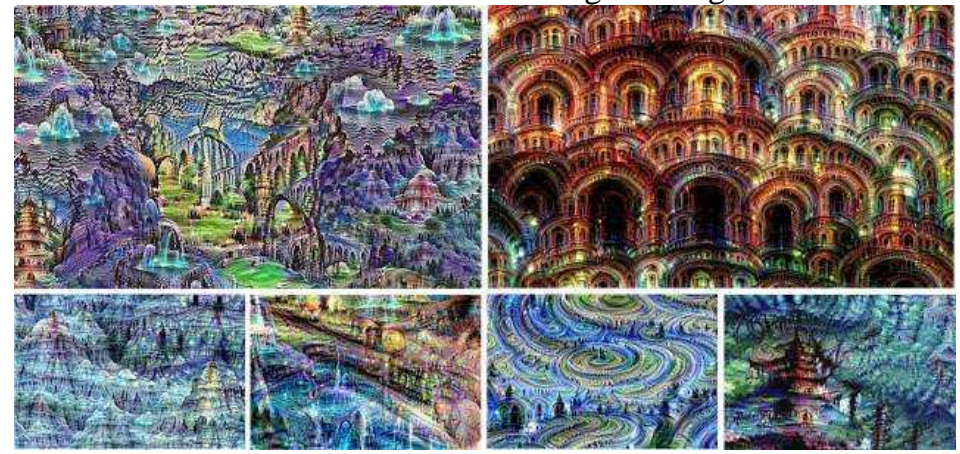

Fig. 4: The work created by the Google Deep Dream program

However, we do not mean that art has no standards. For example, R. Arnheim believes that visual art should conform to Gestalt psychology. This is a kind of "a common core of truth" that makes art associate with anyone at any time and place [14]. The visual psychological forms, like balance, simplification, gestalt, etc. mentioned in Gestalt psychology can indeed become the standard for judging the internal structure of visual art works.

The author believes that different art categories and styles have their own relative standards, and these standards are stable within a certain time and scope. For example, the standard of realist paintings is to realistically represent objects in two-dimensional space, while the standard of modernism is to pursue the sense of reality of the painting plane. From the details of artistic operations, each style has its own standards, such as represent and express, perspective and cavalier perspective, etc., and from the history of whole art, its standard is to pursue artistic reality.

Whether it is CAN or GDD program, it is still only to seek the formal law of visual art through neural networks and Deep Learning. The basic principle is to summarize and calculate the general law on the image of objects (real things, art classics). This kind of law is represented, reorganized and transformed, and then adjusted and evaluated according to the existing artistic styles. Although CAN uses the method of style ambiguity (deviation), its so-called artistic evaluation standard is still derived from the previous artistic styles, and it cannot break through standards and re-adjust standards 
according to the overall law of development of art. But this initiative is what the artist pursues. This requires an overall sense of art and problem consciousness, and artificial intelligence is at least impossible to rise from experience to this overall sense and consciousness. As the CAN designers finally said, artificial intelligence does not really understand art. It knows nothing about the subject and artistic principles. What it does is to learn and imitate human art [3].

\subsection{Art reception in AI art}

The complete art process involves art creation and art appreciation. Although artificial intelligence could not be called creators currently, their works can be appreciated by human. If according to Hegel's theory that artworks need to be instilled with the spirit of human beings, then the works created by artificial intelligence are hard to be called as artworks. However, if we look at from aesthetics of reception, although art lacks intentional subject creators, the works can still be understood and appreciated by human in fact.

From the perspective of Aesthetics of Reception, if a piece of artwork is not appreciated by anyone, it cannot be called a work of art. That is to say, the appreciators are the same as the creators for jointly creating the artwork.

H. Jauss, the founder of aesthetics of reception theory, puts forward the concept of "horizon of expectation" ([Germany]Erwartungshorizont). The concept emphasizes the requirements of the appreciators' own cultural cultivation, taste, experience and ideals. Therefore, different appreciators may make different interpretations and judgments on the same piece of artwork, which is why CAN designers divide testers into several different groups, distinguishing between ordinary lovers and art experts.

S. Fish further emphasizes the reader's experience in the reading process, and believes that this kind of experience can help to complete the meaning of the work. He suggests that reader is a decisive role for the work, because the social meaning, aesthetic value and even the potential meaning of the text need to be realized in the reader's creative reading. From this perspective, artificial intelligence artworks are likely to produce its unique aesthetic value, such as the work of Deep Dream, Aaron and etc. Although we may not like this type of artworks, they are too far away from the art forms we have accepted. Only when we have seen more and more such works and our aesthetic taste are changed, we can interpret and appreciate them. Therefore, if we only consider the results and don't reflect on the makers temporarily, we can't neglect on the aesthetic characters of AI works.

Some people could criticize that the aesthetic dimension of AI works is endowed with by human, the beauty of AI works has no difference with the natural beauty if AI has no purpose to make works. This is hard to argue if we don't discuss the intention of subject.

In addition, art reception also involves the relationship between autonomy and heteronomy in art. Does art bring liberation to people, or will it become a tool for dominating people? W. Benjamin mentioned in The Work of Art in the Age of Mechanical Reproduction, mechanical reproduction technology can reproduce a large number of 
works, which can give the public an opportunity to appreciate the art, thus bringing the opportunity to liberation [15]. Then whether the work made by artificial intelligence is the reproduction of the human artistic style, or has its own unique style, AI can bring cheaper and more convenient works to the public. However, as T. Adorno pointed out, this kind of industrialized artworks can also become a tool of the administrative class to control the public. Humans no longer have the ability to take control of their own aesthetic tastes. All aesthetic and even cognitive ability may be controlled by others, even more boldly, controlled by intelligent machines.

Finally, the art of artificial intelligence also raises a question for our artists, that is, after the emergence of artificial intelligence art, what is the independent value and significance of human art? Since the intelligent agent has been able to massively produce artworks, is it necessary for human beings to create art? The author believes that this highlights the significance of human art to the creator. Art is the need of artists for expression and communication. Art as a human spiritual product, its ultimate goal is to achieve the harmony between human body and mind, between people and people and between people and nature, is to actualize spiritual transcendence. Human beings are to achieve the expansion of the aesthetic mind and ultimate freedom through art. And because of the machine properties of artificial intelligence, it has no body and mind, so it can't touch the core part of art. Therefore, we can predict that after the emergence of artificial intelligence art, human art will pay more and more attention to the liberation and freedom of the creators themselves.

\section{Conclusion}

From this, we can see that although artificial intelligence programs have been able to create works of art with unique styles, this does not mean that they are creative, because creativity is not only the uniqueness and innovation of styles, but also means that this style must be exemplary, and need creation subjects to understand the entire art history, and have independent artistic sense and problem consciousness. Similarly, judging the creativity of art requires the establishment of artistic standards, and the artistic standards are constantly changing with time and space. At present, only from the perspective of appreciation and reception of artworks, the positive meaning and value of artificial intelligence art could be considered. With the emergence of artificial intelligence art, people need rethink the definition of art and the independent value of human art.

However, the author believes that with the advancement of artificial intelligence technology, artificial intelligence may create more and more works with independent aesthetic styles and aesthetic values. Artificial intelligence art is not only for us to appreciate, but also to bring us be closer to the essence and truth of human art.

\section{Acknowledge}

This paper is supported by the National Social Science Fund of China, the name of this project is "The Language Thought in Adorno's Philosophy". No. 16BZX118 


\section{References}

1. Gombrich, E.: The Story of Art. Phaidon Press Ltd, London(2006).

2. Gatys, L., and etc.: "A Neural Algorithm of Artistic Style", https://arxiv.org/pdf/1508.06576v1.pdf , 2015

3. Elgammal, A., and etc.: "CAN: Creative Adversarial Networks Generating 'Art' by Learning About Styles and Deviating from Style Norms”, https://arxiv.org/pdf/1706.07068.pdf, June 23, 2017, pp.1-22

4. Negnevitsky, M.: Artificial Intelligence, A Guide to Intelligent Systems, 2nd edn. Pearson Education Limited, New York (2005)

5. Goodfellow, I., Bengio, Y., Courville, A., Deep Learning. MIT Press, Boston (2016)

6. Boden, M.: "Creativity and artificial intelligence", Artificial Intelligence 103,347-356 (I998).

7. Turing, A.: "Computing Machinery and Intelligence”, Mind. 49, , pp.433-460 (1950).

8. Boden, M.: Artificial intelligence, Academic Press, San Diego(1996)

9. Gips, J., Stiny, G.: “Artificial Intelligence and Aesthetics". IJCAI'75 Proceedings of the 4th international joint conference on Artificial intelligence, vol.1, https://www.ijcai.org/Proceedings/75/Papers/135.pdf ,pp. 907-911

10. Shimamura, A.: "Toward a Science of Aesthetics issues and ideas", In Palmer,S.E(eds.) Aesthetic Science: Connecting Minds, Brains and Experiences. pp.3-28. Oxford University Press, Oxford(2012)

11. Firschein, O., Fischler, M.: "Forecasting and Assessing the Impact of Artificial Intelligence on Society", https://www.ijcai.org/Proceedings/73/Papers/013.pdf, 1973

12. Adorno, T.: Gesammelte Schriften, Band.7: Ästhetische Theorie . Tiedemann, R. unter Mitwirkung von Adorno, G., Buck-Morss, S., Schultz, K. (eds.). Suhrkamp, Frankfurt (2003)

13. Tao, F.: "The Standard of Art: Adorno on Truth Content", Nanjing College of Art, vol. 2, 21-25 (2013)

14. Arnheim, R.: Art and Visual Perception: A Psychology of the Creative Eye, University of California Press, Los Angel(1997)

15. Benjamin, W.: "Das Kunstwerk im Zeitalter seiner technischen Reproduzierbarkeit". Gesammelte Schriften. Band 7. Tiedemann, R., Schweppenhäuser, Hermann.(eds.) ,471508.Suhrkamp, Frankfurt am Main (1991). 